\title{
Component dynamics in nanostructured PI-PDMS diblock copolymers with PI segregated in lamellas, cylinders and spheres
}

Angel Alegria, Reidar Lund, ${ }^{1}$ Fabienne Barroso-Bujans, ${ }^{2}$ Arantxa Arbe, and Juan Colmenero

Polymers and Soft Matter Group. Centro de Física de Materiales, CSIC-UPV/EHU \& Departamento de Física de Materiales, University of The Basque Country.

Po Manuel de Lardizabal 5, 20018 Donostia-San Sebastian (Spain)

\author{
${ }^{1}$ Present address: Dept. of Chemistry, University of Oslo, Postboks 1033, Blindern 0315 Oslo (Norway) \\ ${ }^{2}$ Present address: Donostia International Physics Center, $\mathrm{P}^{\mathbf{o}}$ Manuel de Lardizabal 4, 20018 Donostia-San \\ Sebastian (Spain)
}

\begin{abstract}
The dynamics of the two components of self-assembled diblock polyisoprene (PI) - polydimethylsiloxane (PDMS) copolymers is investigated by means of broadband dielectric spectroscopy. By varying the size of the PDMS blocks, different PI segregation geometries are obtained, namely lamellas, cylinders, and spheres, with typical sizes in the range 6-16 $\mathrm{nm}$. In this way we identify the effects of nano-structure formation on the dielectric relaxation of the polymer components- both the $\alpha$-relaxation of both blocks as well as the normal mode of PI. Two different situations are explored: i) the PDMS dynamics is detected in a temperature range where the PI phase is frozen ii) the PI dynamics is detected in a temperature range where the PDMS phase is highly mobile. Thus, using a single system the similarities and differences between hard- and soft-confinement are investigated.
\end{abstract}

\section{Keywords}

Dielectric relaxation, block copolymers, polymer dynamics, nanostructure, confinement effects

\section{Introduction}

A-B diblock copolymers can self-assemble by forming periodic nanostructures such as spheres, cylinders, gyroids, lamellas, and other complex structures [1-7]. The parameters governing the block segregation are the volume fraction, $f$, the temperature-dependent Flory-Huggins interaction parameter, $\chi$, and the degree 
of polymerization, N. For instance, at high asymmetries, when the fraction of the A block $f_{\mathrm{A}}$ is low, highly curved surfaces are favored leading to cylindrical or spherical A domains. For moderate asymmetries, complex bi-continuous states or gyroid phases are formed and for $f_{\mathrm{A}} \sim 0.5$ lamellar phases occur. A wealth of studies of different block copolymer systems have been presented in the literature. While a useful compilation on the morphology of different A-B block copolymers can be found in e.g. references $[4,7]$, here we will briefly mention two selected systems. Polystyrene-polyisoprene (PS-PI) is a classical system that has been extensively investigated and used as a model system for fundamental studies for block copolymer self-assembly [4,8-10]. Although PS-PI model block-copolymers are simple to make through living anionic polymerization, the system is characterized by relatively low $\chi$ parameters $(\chi \sim 0.06$ at $298 \mathrm{~K})$ [11], which demands large molecular weights in order to obtain well-segregated structures. An attractive alternative model system was introduced by Almdal, Bates and coworkers [12] who prepared well-defined structures of poly(isoprene)-poly(dimethylsiloxane) (PI-PDMS) and of its hydrogenated derivative, poly(ethylene-alt-propylene)-poly(dimethylsiloxane) (PEP-PDMS) by sequential living anionic polymerization and ring opening polymerization. In contrast to PS-PI, this polymer system exhibits very low glass transition temperature and relatively high $\chi$-parameter $(\chi \sim 0.24$ and 0.13 for PEP-PDMS and PI-PDMS respectively at $298 \mathrm{~K}$ ) [13,14]. As shown in a series of publications, this system displays well-defined morphologies which can be further ordered at room temperature using mechanical shear [12,13] leading to long-range ordered almost single crystal structures. The tunable morphologies of block copolymers provide a variable range of geometrical constraints for fundamental studies devoted to the polymer dynamics under confinement [15-17]. In this context, the PI dynamics in segregated phases formed in PI-PDMS block copolymers have been subjected to recent studies by broadband dielectric [16,18] and neutron spin echo spectroscopy [17]. Block copolymers with PI volume fractions, $f_{\mathrm{PI}}$, in the range of $0.16-0.19$ form hexagonally ordered PI cylinders or spheres in a continuous matrix of PDMS polymer. PDMS is a highly flexible polymer with a low glass transition temperature and thus can be considered as a soft confinement for the PI domains. However, upon cooling PDMS blocks crystallize limiting the range where the PI dynamics can be investigated.

In the present study, we explore a wider range of compositions and temperatures in PI-PDMS block copolymers. In this way we found that it is possible to investigate the effect of the interface geometry on the dynamics of both copolymer components in a single system by dielectric relaxation. Moreover, the effect of confinement on the PDMS crystallization also allowed us to explore the whole temperature 
range where the dielectric relaxation of both components occurs. Consequently, here we present a comprehensive dielectric study of the effects of copolymer self-assembly on the dynamics of both components. Particularly, we show how in the low temperature range the PDMS segmental dynamics is affected by the presence of glassy PI segregated phase. In addition, at higher temperatures, we show how the PI dynamics in the segregated phase is affected by the presence of highly fluctuating interfaces. The paper is organized as follows: first we briefly describe the polymers investigated and the methods used for structural and thermal characterization. The dielectric relaxation experiments and the analysis procedure are presented in detail. Afterwards, we show the characteristics of the nano-structures detected in the copolymers and the thermal behavior of the samples investigated. After this complete characterization, we present and discuss the dielectric relaxation results obtained in different temperature ranges, which reflect the dynamic behavior of the two polymer components: the PDMS segmental dynamics and the PI segmental and chain dynamics. Finally, we present the main conclusions of this work.

\section{Experimental}

PDMS homopolymers with number-average molecular weights, $M n$, of 21 and $3.5 \mathrm{~kg} / \mathrm{mol}$ and polydispersity index of 1.1, were purchased from Polymer Source inc.

PI and PI-PDMS block copolymers were synthesized by anionic living polymerization as described in the Supplementary Information of reference [16]. The molecular weight and molecular weight distribution of the synthesized polymers are summarized in Table 1 . Note that the value of $\mathrm{Mn}$ (in $\mathrm{kg} / \mathrm{mol}$ ) of each component of the block copolymer is indicated in the copolymer name (e.g. PI4-PDMS3.5 is composed by PI of $4 \mathrm{~kg} / \mathrm{mol}$ and PDMS of $3.5 \mathrm{~kg} / \mathrm{mol})$.

Table 1 Molecular weight characteristics of the synthesized polymers.

\begin{tabular}{lccccc}
\hline $\begin{array}{l}\text { Block } \\
\text { Copolymer }\end{array}$ & $\begin{array}{c}\text { Mn } \\
(\mathrm{kg} / \mathrm{mol})\end{array}$ & Mw/Mn & $\begin{array}{c}\mathrm{Mn} \\
(\mathrm{kg} / \mathrm{mol})\end{array}$ & $\mathrm{Mw} / \mathrm{Mn}$ & $f_{\mathrm{PI}}{ }^{*}$ \\
& $(\mathrm{PI})$ & $(\mathrm{PI})$ & $\begin{array}{c}\text { (PI-PDMS }) \\
(\mathrm{PI}-\mathrm{PDMS})\end{array}$ & \\
\hline PI4-PDMS3.5 & 4.3 & 1.04 & 7.8 & 1.03 & 0.58 \\
PI4-PDMS4 & 4.3 & 1.04 & 8.4 & 1.03 & 0.54 \\
PI4-PDMS23 & 4.3 & 1.03 & 27.8 & 1.03 & 0.17 \\
PI4-PDMS32 & 4.4 & 1.03 & 36.4 & 1.04 & 0.11 \\
\hline *volume fraction of PI calculated using $\rho_{\mathrm{PI}}=0.9 \mathrm{~g} / \mathrm{cm}^{3}$ and $\rho_{\mathrm{PDMS}}=0.99 \mathrm{~g}^{2} \mathrm{~cm}^{3}$.
\end{tabular}




\section{Small-angle X-ray Scattering}

The structure of the block copolymers was investigated in a Rigaku SAXS instrument. The $4.5 \mathrm{~m}$ long instrument consists of a MM002+ micro focus source, a 2D-200X gas detector and a 3 pin hole collimation system. The temperature was controlled within $\pm 0.2 \mathrm{~K}$ using a Linkam stage. The data were normalized with respect to transmission and thickness, the background was subtracted and brought to an absolute scale in units of $\mathrm{cm}^{-1}$ by measuring the scattering of water in capillaries that allow quantitative background subtraction.

\section{Differential scanning calorimetry}

Differential scanning calorimetry (DSC) measurements were carried out on $\sim 10 \mathrm{mg}$ specimens placed in sealed aluminum pans using a Q2000 TA Instruments apparatus in both standard and temperaturemodulated (TM) modes. All samples were first cooled rapidly to $120 \mathrm{~K}$. Next, both standard and TMDSC experiments were performed. Standard DSC measurements were conducted at $20 \mathrm{~K} / \mathrm{min}$ heating rate. TM-DSC experiments were done using an average heating rate of $2 \mathrm{~K} / \mathrm{min}$ and a sinusoidal temperature oscillation of $0.5 \mathrm{~K}$ amplitude and 60-second modulation period. A helium-flow rate of 25 $\mathrm{ml} / \mathrm{min}$ was used for thermalization.

\section{Broadband dielectric spectroscopy}

The complex dielectric permittivity, $\mathcal{E}^{*}\left[\varepsilon(\omega)^{*}=\mathcal{E}^{\prime}(\omega)-i \mathcal{E}^{\prime \prime}(\omega)\right]$, was measured over a wide frequency range, $\omega / 2 \pi \cdot 10^{-2}-10^{7} \mathrm{~Hz}$, using a Novocontrol high-resolution dielectric analyzer (Alpha-S analyzer). The samples were prepared directly on $30 \mathrm{~mm}$ diameter gold-plated electrodes and left to dry at room temperature in a vacuum oven in order to remove any rest of solvent. Next, the samples were squeezed using upper gold-plated electrodes of $20 \mathrm{~mm}$ diameter, forming finally parallel plate capacitors by using finely cut $0.1 \mathrm{~mm}$ thick star-shaped Teflon pieces as spacer. These sample capacitors were kept under vacuum before use to remove any trapped bubble. The data were collected isothermally and the temperature was controlled within $\pm 0.1 \mathrm{~K}$ using a Novocontrol Quatro cryostat that uses a continuous nitrogen-jet flow.

In order to determine the characteristic time scales for the different relaxation processes the peak relaxation time, $\tau_{m}$, was determined from the peak frequency, $\omega_{\max }$, of the corresponding loss component, i.e. $\tau_{m}=1 / \omega_{\max }$. When several relaxation components overlapped partially, the data were fitted to the 
superposition of two Havriliak-Negami (HN) phenomenological relaxation functions [19,20]. The dielectric loss equation corresponding to the Havriliak-Negami function reads:

$\varepsilon^{\prime \prime}(\omega)=\operatorname{Im}\left\{\frac{-\Delta \varepsilon}{\left[1+\left(i \omega \tau_{H N}\right)^{\alpha}\right]^{\gamma}}\right\} \quad$ eq. 1

where $\Delta \varepsilon$ corresponds to the relaxation strength, $\alpha_{H N}$ and $\gamma_{H N}\left(\alpha_{H N}, \gamma_{H N}<1\right)$ are parameters determining the peak shape, and the relaxation time $\tau_{H N}$ is related to the peak relaxation time as [20]:

$\tau_{m}=\tau_{H N}\left[\sin \frac{\alpha_{H N} \pi}{2+2 \gamma_{H N}}\right]^{-1 / \alpha_{H N}}\left[\sin \frac{\alpha_{H N} \gamma_{H N} \pi}{2+2 \gamma_{H N}}\right]^{1 / \alpha_{H N}}$ eq. 2

\section{Sample characterization}

Small angle $X$-ray scattering

Fig. 1 shows the SAXS patterns recorded at room temperature for the different copolymers. They directly evidence the effect of the size of the PDMS block on the nanostructure and particularly on the nanosegregation geometry. In agreement with the expectations of the theoretical phase diagram of diblock copolymers [2], lamella structures are found for the shortest PDMS blocks with a size similar to that of the PI one (PI4-PDMS3.5 and PI4-PDMS4). By increasing the PMDS block-size (PI4-PDMS23), PI becomes segregated into hexagonally packed cylinders, and by increasing further the PDMS block (PI4PDMS32), PI segregates into spheres forming a BCC lattice. The solid lines in Fig. 1 stand for the fitting curves resulting with the abovementioned geometrical models by using "Scatter", a software developed by S. Förster and L. Apostol [21]. In these models, contributions to the scattering function from both structure and form factors are considered [22]. The main structural characteristics so obtained for the nano-segregated block-copolymers are summarized in Table 2. 


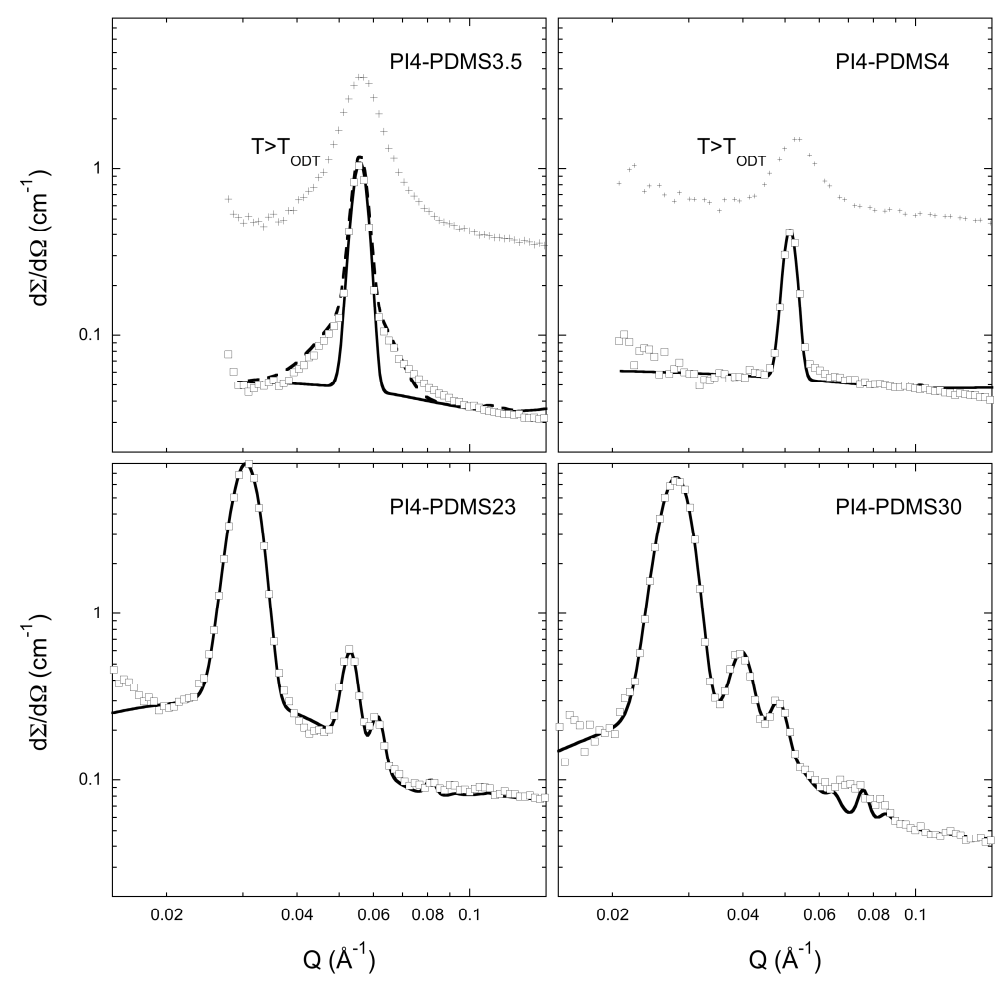

Fig. 1 SAXS patterns obtained at room temperature on the PI-PDMS copolymers investigated. The lines correspond to the fitting to simple lattice models (see text). The crosses in the upper frames correspond to one decade vertically shifted SAXS patterns obtained at temperatures $20 \mathrm{~K}$ above the corresponding ODT, namely $330 \mathrm{~K}$ and $120 \mathrm{~K}$ for PI4-PDMS3.5 and PI4-PDMS4 respectively.

The copolymer with shortest PDMS block (PI4-PDMS3.5) shows a broad component suggesting the presence of a significant amount of defects. By reducing the domain size of the crystal to only $\sim 3$ times the lattice constant, the data can be best fitted to the geometrical model used (dashed line) indicating a contribution of $\sim 30 \%$ of defective structures. For the PI4-PDMS4 copolymer, a weaker component of this type could also exist but with an upper bound contribution of $\sim 10 \%$.

Table 2 Characteristics of the copolymer nanostructures.

\begin{tabular}{ccc} 
PI segregated phase & $\begin{array}{c}\text { size } / \mathrm{nm} \\
\text { (thickness or diameter) }\end{array}$ & lattice-constant/nm \\
\hline lamella & 6.5 & 11.3 \\
lamella & 6.8 & 12.5 \\
cylinder (HPC) & 9.0 & 23.5 \\
sphere (BCC) & 16 & 30.5 \\
\hline
\end{tabular}


Temperature dependent experiments showed that the geometry of the nanostructured systems remains unaltered over the whole temperature range investigated (140-400 K) with only minor changes in size, except for the lamella nanostructures. Rheological experiments in Fig. 2 showed a dramatic reduction of the shear modulus at $310 \mathrm{~K}$ for PI4-PDMS3.5 and $370 \mathrm{~K}$ for PI4-PDMS4, indicating the existence of an order-to-disorder transition (ODT). The lower ODT temperature for PI4-PDMS3.5 is in qualitative agreement with the more imperfect self-assembled structure as discussed above. SAXS patterns of Fig. 1 also confirm that above the ODT there is an absence of sharp diffraction peaks.

The detection of the ODT in PI4-PDMS3.5 and PI4-PDMS4 is in qualitative agreement with theoretical expectations. However, the observed ODT temperatures are higher than those expected from the theory taking into account the literature values [23] for the interaction parameter between PI and PDMS: $\chi=$ $43.6 / T-0.01$. In this way, we obtain $\chi N=13$ for PI4-PDMS4 and $\chi N=12$ for PI4-PDMS3.5 at the ODT temperatures, whereas, according to the Leibler mean field model [24] for symmetric diblock copolymers, a second order ODT should occur for $\chi N<10.5$. Although this value seems to be incompatible with our observed ODT, it should be noted that, for short block copolymers and intermediate $\chi$-values (intermediately segregated systems), a fluctuation-induced first-order transition was predicted [2]. Therefore, there might be a dependence of the ODT with the molecular weight. If we calculate the $(\chi N)_{\mathrm{ODT}}$ value according to $(\chi N)_{\mathrm{ODT}}=10.495+41.022 / \mathrm{N}^{1 / 3}[25]$ and the molecular volumes of each block, we obtain that $(\chi N)_{\mathrm{ODT}}$ for PI4-PDMS3.5 is 19 and that for PI4-PDMS4 is 18.8. These values, higher than those obtained above $(\chi N=12$ and 13), explain why we observed an ODT in our symmetric PI-PDMS diblocks. Notwithstanding the above, according to these predictions, the ODT should occur at about 240 K and 255 K for PI4-PDMS3.5 and PI4-PDMS4, respectively, which are by far lower than what we found experimentally ( $310 \mathrm{~K}$ and $370 \mathrm{~K})$. It must be realized that mapping between experimental and theoretical $\chi$ values is not easy -also because it scales directly with the lattice site volume, which, in addition, is not determined trivially by experiments [26]. In fact, a simple scaling of the $\chi$ values by a factor of 1.35 gives a much better prediction for ODT temperatures. A similar factor (1.2) was also applied by Morse and coworkers [23] to obtain a better agreement of their data. However, our calculations with 1.35 factor showed only $20 \mathrm{~K}$ of difference between the experimental ODT temperatures of PI4-PDMS3.5 and PI4PDMS4. Thus, the rather dramatic effect of the chain length on ODT obtained experimentally remains unclear and should be investigated in more detail. 


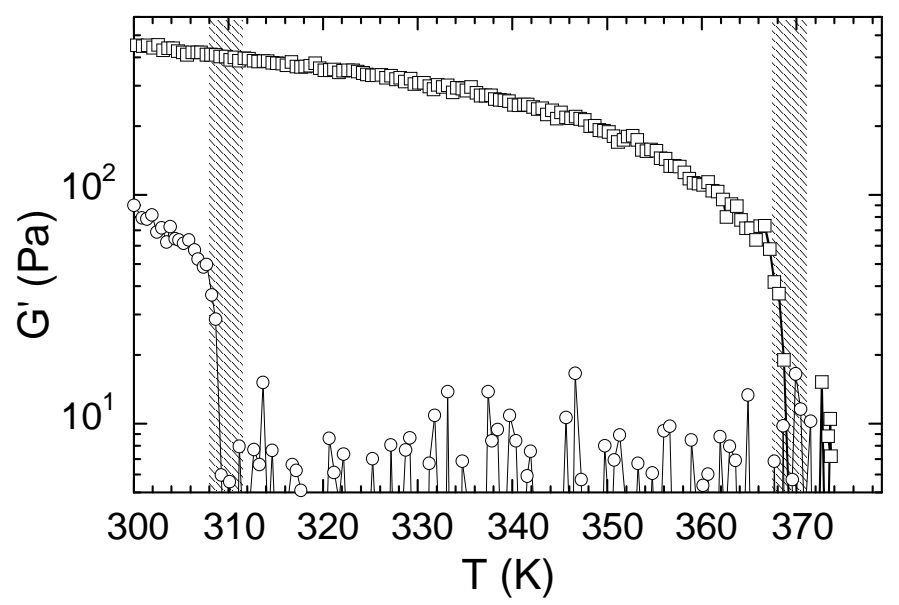

Fig. 2 Temperature dependence of the shear storage modulus of the two symmetric diblock copolymers: PI4-PDMS4 (squares), PI4-PDMS3.5 (circles) [27]. The shadowed areas correspond to the temperature ranges were the order-disorder-transition is identified.

\section{Differential scanning calorimetry}

Fig. 3 presents the thermal behavior of the copolymers and the corresponding homopolymers as determined by DSC on samples quenched from room temperature to avoid any eventual crystallization of PDMS. Fig. 3a shows the effect of the molecular weight of PDMS homopolymer on both the glass transition and the subsequent cold-crystallization. At temperatures above $220 \mathrm{~K}$, complex melting processes are also detected. The effect of molecular weight on the glass transition process is just a minor temperature shift, as previously reported [28]. However, both the cold-crystallization temperatures and the melting peaks depend markedly on the molecular weight. In the asymmetric copolymers (Fig. 3b) the glass transition and melting of PDMS remains nearly unaffected but the cold-crystallization depends noticeably on the nanostructure. This situation is drastically different for the symmetric copolymers, where the glass transition of PDMS is clearly shifted to higher temperatures and no signature of coldcrystallization (or melting) is detected (see Fig. 3c). Remarkably, these are the only cases where the glass transition of PI blocks could be detected although with a shape that is quite different from that of the PI homopolymer. Particularly, the step in heat flow is markedly more extended in the low temperature side. Unfortunately, there is no clear signature of the glass transition of PI in the rest of block copolymers (see inset in Fig. 3b), most likely because of the dominating exothermic crystallization process of the PDMS matrix over the glass transition of minority PI phase. A summary of the temperatures characterizing the thermal behavior of the samples investigated is shown in Table 3. 

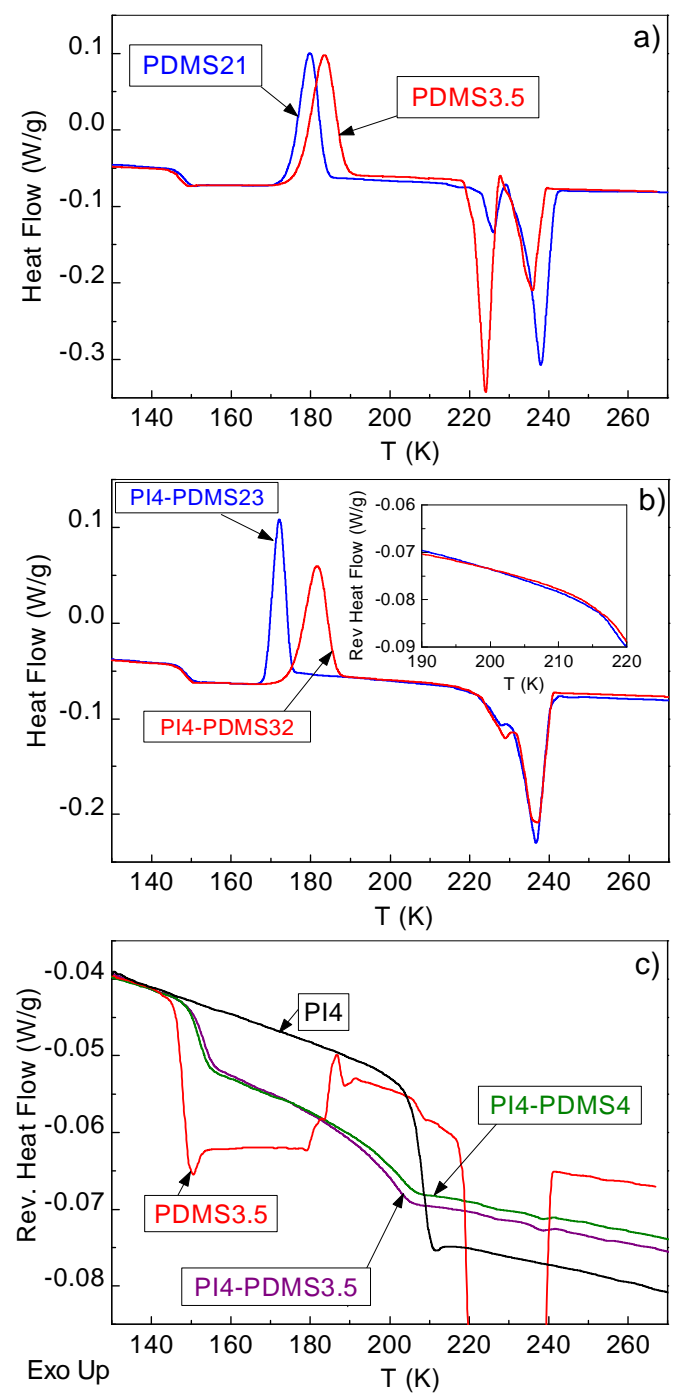

Fig. 3 DSC (a,b) and TM-DSC (c and inset of b) experiments recorded during heating (see experimental part for details). Inset in frame $b$ shows the temperature range where the glass transition of the PI phase is expected to occur.

Table 3 Temperatures characterizing the main thermal features of the investigated samples.

\begin{tabular}{lccc}
\hline & $\begin{array}{c}\mathrm{T}_{\mathrm{g}}(\mathrm{PDMS}) / \mathrm{K} \\
\text { medium (onset) }\end{array}$ & $\begin{array}{c}\mathrm{T}_{\mathrm{g}}(\mathrm{PI}) / \mathrm{K} \\
\text { medium (onset) }\end{array}$ & $\begin{array}{c}\mathrm{T}_{\mathrm{C}}(\mathrm{PDMS}) / \mathrm{K} \\
\text { cold-cryst. peak }\end{array}$ \\
\hline PI4 & - & $208.3(205.6)$ & - \\
PDMS3.5 & $147.9(146.2)$ & - & 183.6 \\
PDMS21 & $149.6(148.2)$ & - & 179.8 \\
PI4-PDMS3.5 & $153.1(149.9)$ & $201.6(195.7)$ & - \\
PI4-PDMS4 & $151.9(149.6)$ & $202.7(196.9)$ & - \\
PI4-PDMS23 & $149.4(147.2)$ & undetected & 172.1 \\
PI4-PDMS32 & $149.2(147.5)$ & undetected & 181.6 \\
\hline
\end{tabular}




\section{Results and discussion}

According to the DSC results of both symmetric copolymers, the effects of the nanostructure on the dynamics of the block components can be investigated by dielectric spectroscopy without the influence of PDMS crystallization. On the contrary, in asymmetric copolymers, the crystallization of the PDMS block interferes in the dielectric response of minority PI phase in the temperature range between $170 \mathrm{~K}$ and 230 K. Notwithstanding the above, the segmental dynamics of the PDMS phase below $170 \mathrm{~K}$ and the segmental and normal mode relaxation of the PI phase above $230 \mathrm{~K}$ are well accessible by dielectric relaxation experiments. In addition, the effects of the ODT on the dielectric relaxation of the symmetric copolymers can also be studied.

\section{Segmental dynamics of PDMS}

Fig. 4 shows a comparison of the loss curves obtained at a relative low temperature where the only contribution to the dielectric relaxation comes from the PDMS phase. Note that at this temperature, well below the $\mathrm{T}_{\mathrm{g}}$ of PI homopolymer, the segregated PI phase is frozen. According to this, the vertical axis was normalized by the volume fraction of PDMS. At this temperature, the rather prominent $\alpha$-relaxation of PDMS is detected at frequencies around $100 \mathrm{kHz}$ with a sharp decrease in the dielectric losses at lower frequencies, as commonly observed in simple glass forming materials. The loss peak position depends slightly on the molecular weight, but the peak shape and the peak intensity remain nearly the same. Even for the copolymers rich in PDMS, the dielectric loss peak is significantly affected with respect to that of the homopolymer, in particular in the low frequency behavior. From the data it is evident that a tail extended towards the low frequency region emerges and ends in a rather flat, almost frequencyindependent plateau. In addition, the position of the loss peak is slightly shifted towards lower frequencies, although the shape of the peak at higher frequencies seems to be unaltered. For symmetric copolymers, this situation changes. The loss peak frequency shifts markedly towards lower values by about one decade. In addition, the tail extended to low frequencies becomes much more pronounced and the lowest frequency loss level is much higher.

The changes observed in peak position are congruent with the presence of a frozen phase, to which the PDMS blocks are attached, which inevitably slows down the molecular mobility of the segments close to the anchoring point. However, the change of the peak frequency is about the same (within uncertainties) for the two asymmetric copolymers, which still remain distinguishable from that of the corresponding 
homopolymer. As the length of the PDMS block is reduced, the influence of anchoring is higher, as it involves a higher fraction of PDMS segments. This effect is evidenced by the more pronounced tail and much higher loss level at low frequencies. In fact, it has been proposed [29,30] that the anchored segments in the interface are at the origin of the losses detected at the lowest frequencies. A result supporting this idea, is the similarity between the low frequency tails in the copolymers as compared to that of cold-crystallized PDMS in a lamellar-like nanostructure with alternating amorphous and crystalline regions [31]. The dielectric losses of cold-crystallized PDMS21 are represented by dashed lines in Fig. 4. It is clear that in all copolymers of Fig. 4, the behavior observed at the lowest frequencies resembles that corresponding to cold-crystallized PDMS. In such a highly crystalline sample (degree of crystallinity close to $40 \%$ ), most of the remaining mobile PDMS segments would be very much influenced by the nearby anchors to the PDMS crystallites.

In all the copolymers investigated here, the overall PDMS segmental dynamics is slower than that in pure PDMS. However, it has been found that when the segregated PDMS phase is fully surrounded by a frozen PS matrix [30], the dielectric $\alpha$-relaxation in the segregated PDMS phase appears at higher frequencies than that in the homopolymer. This effect was attributed to the packing restrictions associated to the difference in the thermal expansion between the segregated phase and the surrounding frozen matrix. This result was accompanied by the inability of the PDMS phase to crystallize. In our PI-PDMS copolymers, these packing restrictions do not exist since the PDMS phase is not entirely surrounded by a frozen PI matrix, although we observed the inability of the PDMS phase to crystallize in the lamella phases. In this case, it is likely that the monomeric PDMS diffusion is reduced due to the overall dramatic slowing down of the segmental PDMS dynamics. 


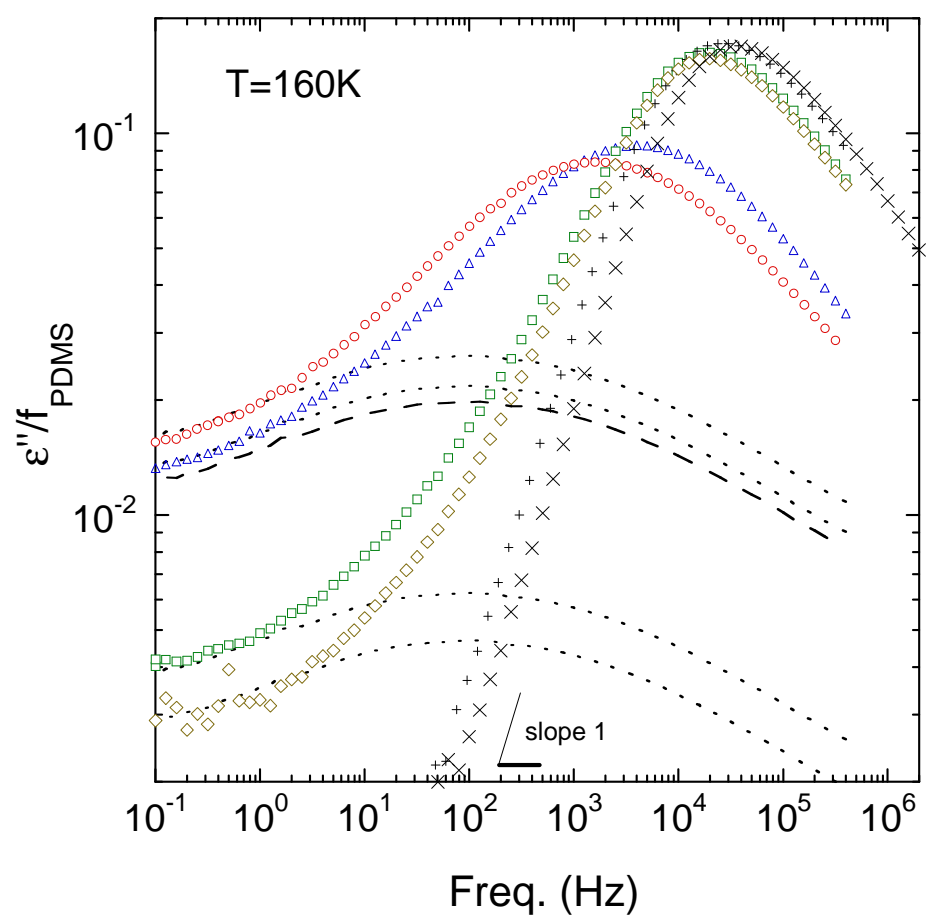

Fig. 4 Dielectric relaxation showing the PDMS segmental dynamics for the samples investigated: PDMS3.5 (x), PDMS21 (+), PI4-PDMS4 (triangles), PI4-PDMS3.5 (circles), PI4-PDMS23 (squares), and PI4-PDMS32 (diamonds). The long-dashed lines correspond to the dielectric relaxation of coldcrystallized PDMS21. Short-dashed lines are the vertically shifted data of cold-crystallized PDMS21 overlapping the copolymer data at the lowest frequency range.

Fig. 5 shows the peak relaxation times characterizing the PDMS segmental dynamics in an Arrhenius representation where the temperature axis is scaled by the glass transition temperature determined by DSC for PDMS homopolymers of similar molecular weights. After scaling, the data of the two PDMS homopolymers superimpose each other and the remaining differences with the copolymers have to be undoubtedly attributed to the effects of the nanostructure. Here the deceleration of the PDMS segmental dynamics in all the PI-PDMS copolymers is evident. This effect is small for the asymmetric copolymers but it is very dramatic for the symmetric copolymers. The temperature dependence of the PDMS segmental dynamics times can be well described by the Vogel-Fulcher-Tamman (VFT) equation [32]:

$$
\tau(T)=\tau_{\infty} \exp \left(\frac{D T_{0}}{T-T_{0}}\right) \quad \text { eq. } 3
$$

where $\mathrm{D}$ is related to the so called 'dynamic fragility' introduced by Angell [33], $\tau_{\infty}$ is a typical vibrational time and $T_{0}$ is the temperature where the relaxation time tends to diverge. The values of the fit parameters 
are summarized in Table 4. Note that good descriptions are obtained by allowing only $T_{0} / T_{g}$ to depend on the copolymer. From the VFT fits it is possible to evaluate the so-called BDS dynamic glass transition temperature, $T_{g B D S}$, as the temperature at which the dielectric relaxation times corresponds to a fixed value. For the PDMS homopolymers, we found that $\tau=1$ s provides a $T_{g B D S}$ value equal to that obtained by DSC (at the inflection point, see values in Table 3). The values calculated in the same way for the copolymers allow us to account for the observed increase in the DSC glass transition temperature of PDMS with respect to the homopolymer. For the two symmetric block copolymers, $T_{g B D S}$ increases by about 2.6 K and 1.7 K for PI4-PDMS3.5 and PI4-PDMS4, respectively. These differences are smaller than those reported in Table 3 for the corresponding calorimetric $T_{g}$ values, which would be due to the fact that the loss peak of the symmetric copolymers is asymmetric with a tail extended towards lower frequencies. Consequently, the loss peak frequency characterizes the segments moving faster than average, in contrast to the homopolymer case. The situation for the asymmetric copolymers is different since most of the PDMS segments are free of the direct influence of anchoring to the glassy PI phase and therefore there is only a tiny fraction that slows down. As a result, there is a nearly undetectable change in the calorimetric $T_{g}$ of the PDMS phase with respect to the corresponding homopolymer.

When considering the heterogeneity of the PDMS segmental dynamics as detected by the asymmetric shape of the dielectric loss peaks, we find that the high frequency part of the loss peak remains similar in all samples and that the effects are prominent mainly (depending on the PDMS volume fraction) at low frequencies. This suggests a rather homogeneous dynamics far from the interfaces but a minor, although detectable, slowing-down of the segmental mobility close to the boundaries. As already commented, the presence of a rigid interface would be responsible of the relaxation extended to very low frequencies, whereas a 'transition region' between these two limiting situations would account for the intermediate frequency range. 


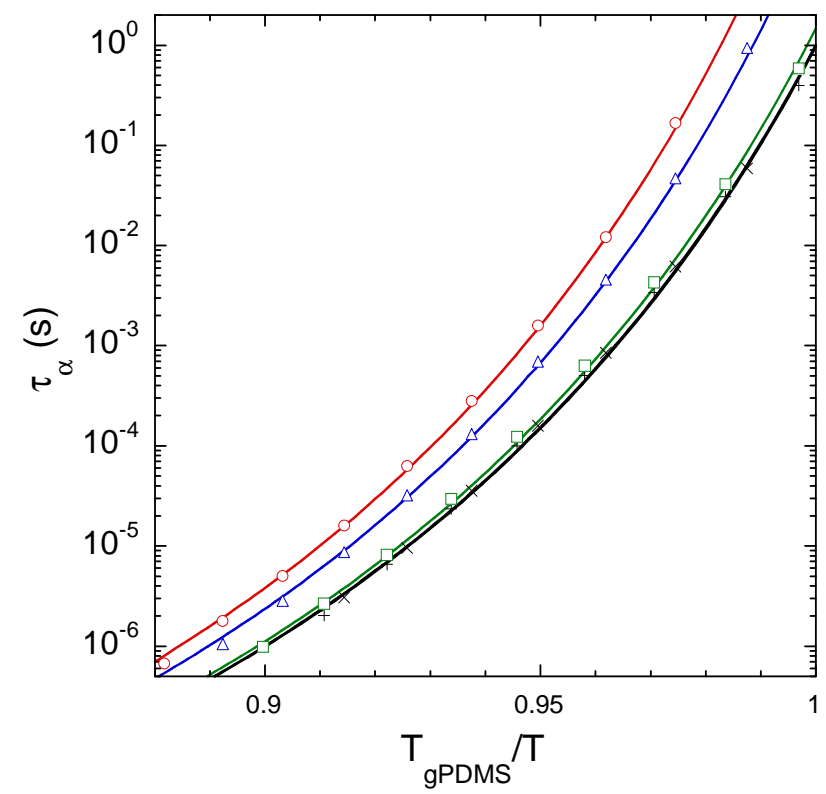

Fig. 5 Temperature dependence of the PDMS segmental dynamics for the samples investigated: PDMS3.5 (x), PDMS21 (+), PI4-PDMS4 (triangles), PI4-PDMS3.5 (circles), PI4-PDMS23 (squares). Data for PI4-PDMS32 are indistinguishable from that of PI4-PDMS23, and therefore they are not represented. The fitting lines were obtained by using the VFT equation (see text).

The anchoring effects on the PDMS segmental dynamics reported herein are similar to those observed in PS-PDMS diblock copolymers, where the PDMS segregates into different geometries- lamellas, cylinders, and spheres $[29,30]$. In the latter cases, the rather high glass transition temperature of PS ensures a frozen interface. The lowest frequency part of the PDMS $\alpha$-relaxation losses behaves similarly to that of cold-crystallized PDMS where the remaining amorphous phase is mainly formed by PDMS subchains anchored to the crystallites.

Table 4 Parameters describing the temperature dependence of the PDMS segmental relaxation time.

\begin{tabular}{lllll}
\hline & $\tau_{\infty P D M S} / \mathrm{s}$ & $D_{P D M S}$ & $T_{0_{-} P D M S} / T_{g D S C \_P D M S}$ & $T_{g B D S} / \mathrm{K}$ \\
\hline PDMS3.5 & $4.35 \mathrm{e}-13(*)$ & 3.81 & 0.8820 & 147.9 \\
PDMS21 & $4.35 \mathrm{e}-13(*)$ & 3.81 & 0.8818 & 149.6 \\
PI4-PDMS3.5 & $4.35 \mathrm{e}-13(*)$ & $3.81\left(^{*}\right)$ & 0.8974 & 150.5 \\
PI4-PDMS4 & $4.35 \mathrm{e}-13(*)$ & $3.81\left(^{*}\right)$ & 0.8920 & 149.6 \\
PI4-PDMS23 & $4.35 \mathrm{e}-13(*)$ & $3.81(*)$ & 0.8833 & 149.8 \\
PI4-PDMS32 & $4.35 \mathrm{e}-13(*)$ & $3.81\left(^{*}\right)$ & 0.8834 & 149.8 \\
\hline
\end{tabular}

(*) Values fixed in the fitting procedure 
The analysis of the intensity of the dielectric losses in the low frequency range allows us to have a rough estimate of the size of the region where PDMS segments become strongly affected by anchoring. The values obtained in this way are compatible with a layer of thickness of about 1-2 nm, in agreement with similar estimates on PS-PDMS copolymers [29,30] and with recent findings for the effect of interface on packing density [34]. This thickness range is slightly larger than that expected from thermodynamic arguments [35] for the composition gradient at the interface. Nevertheless, the effects of anchoring on the PDMS segmental mobility can be also extended to larger distances from the interface since the peak relaxation time for the lamella PDMS phases having a thickness of more than $6.5 \mathrm{~nm}$ is about 10 times longer than in PDMS homopolymer. It is also noteworthy to say that even for the more asymmetric copolymers, where the PDMS volume to surface ratio can be estimated to be about $20 \mathrm{~nm}$, the shift of the loss peak position is still detectable.

The general effect of temperature on the shape of the PDMS $\alpha$-relaxation loss peaks is to reduce the low frequency broadening. This, together with the fact that the main parameter of the VFT equation affected by the nano-structure is $T_{0}$, implies that the broadening can be parameterized in terms of a corresponding distribution of $T_{0}$ values [29]. In this framework, all the relaxation times of a possible distribution would approach to each other as the temperature increases, resulting in a more homogeneous segmental dynamics at high temperature.

\section{Segmental Dynamics of PI blocks}

Fig. 6 shows the dielectric losses for the symmetric diblock copolymers at $213 \mathrm{~K}$. This temperature is a few degrees above the glass transition of PI homopolymer, and therefore, the detected loss peaks correspond to that of the $\alpha$-relaxation of the PI phases. Consequently, the vertical axis in this Fig. has been normalized to the PI volume fraction of the sample to facilitate the comparison with the data of PI homopolymer at similar temperatures. We should recall that in the case of the asymmetric copolymers the PDMS phase is semicrystalline and the PI contribution cannot be identified at these temperatures. However, for the symmetric copolymers, the main contribution of the amorphous PDMS occurs at much higher frequencies in this temperature range. Nevertheless, a flat "background-like" contribution can be noted for the two copolymers. This is most likely associated to the relatively slow PDMS segments directly influenced by anchoring to the PI phase. 
From Fig. 6, it is clear that the peak appears in the copolymers at a frequency about one decade higher than that of the $\alpha$-relaxation peak of PI homopolymer at the same temperature. However, the peak positions match quite well if the comparison is made with the $\alpha$-relaxation peak of PI homopolymer at a temperature $5 \mathrm{~K}$ above. In addition to the shift in peak position, the PI $\alpha$-relaxation in the copolymers is markedly broader than that in the homopolymer. It is noteworthy that the broadening occurs mainly to high frequencies, as it would be expected from the low-temperature-extended calorimetric glass transition. When we compare the relaxation of the two symmetric copolymers, it is found that the loss peak position is more affected in the PI4-PDMS3.5 and that it presents a more extended high frequency tail.

At temperatures higher than the melting temperature of PDMS ( $\mathrm{T}>230 \mathrm{~K}$ ), the PDMS phase becomes fully amorphous also in the asymmetric copolymers and the PDMS dielectric losses occur only at much higher frequencies. Consequently, there is not detectable contribution from PDMS in the explored frequency range, other than the relative low conductivity contributions observed at the lowest frequencies (see Fig. 7). In this figure, the dielectric losses measured in all the diblock copolymers at $243 \mathrm{~K}$ are shown in comparison with that of PI homopolymer at $248 \mathrm{~K}$. Again, the vertical axis is scaled to the PI volume fraction. At this high temperature, the PI $\alpha$-relaxation peak for all the copolymers occurs at about $200 \mathrm{kHz}$. Remarkably, the dielectric normal mode relaxation originated from the fluctuations of the endto-end PI chain vector is also well detected at this temperature at low frequencies.

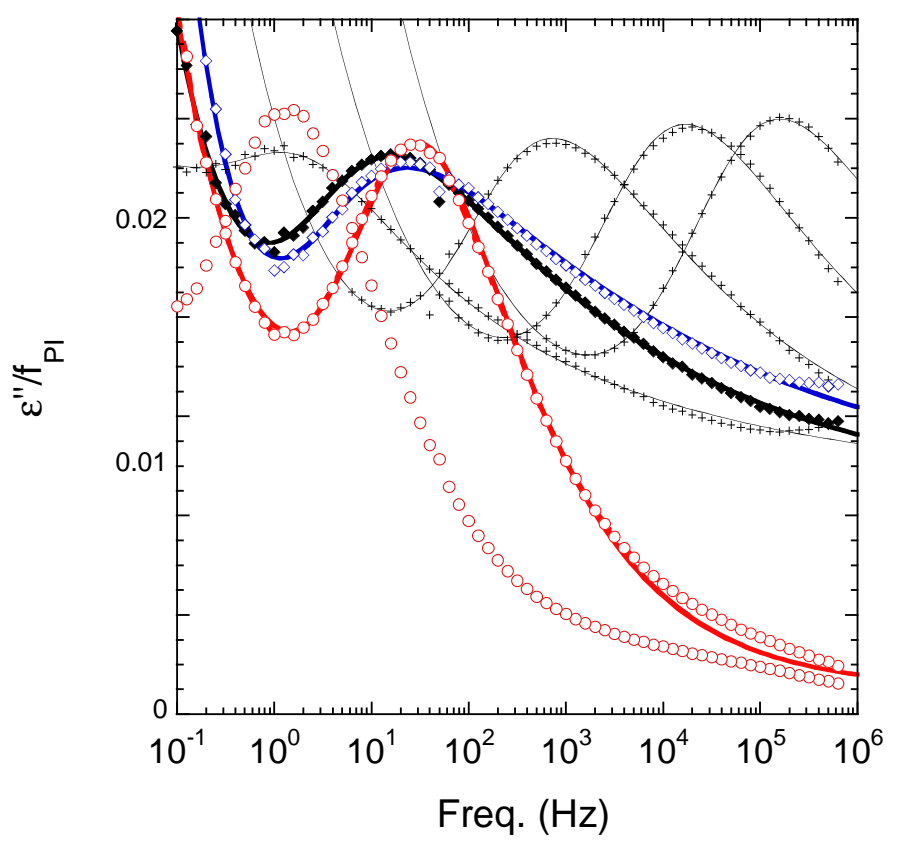


Fig. 6 Dielectric relaxation at $213 \mathrm{~K}$ showing the segmental dynamics of PI for the symmetric copolymers investigated: PI4-PDMS4 (filled diamonds), PI4-PDMS3.5 (empty diamonds). (+) correspond to data collected at other temperatures (5 K step) for PI4-PDMS4. PI4 data (circles) at $243 \mathrm{~K}$ and $248 \mathrm{~K}$ are also shown for comparison. The lines are fitting curves obtained as described in the text.

Fig. 8 shows the characteristic times of the PI segmental dynamics in an Arrhenius representation where the temperature is scaled using the $T_{g}$ value of the reference PI homopolymer as determined by DSC. The relaxation times for the PI component in the segregated phase are smaller than those in the homopolymer. Moreover, there are almost no differences between the values for the copolymers despite the existence of different geometries for the segregated PI phase - at least in the temperature range where the PI $\alpha$ relaxation can be experimentally determined for all the copolymers, i.e. above $230 \mathrm{~K}$. We recall that for all the copolymers the PI chains are virtually identical. Taking this into account, the origin of the acceleration of the segmental dynamics would be related to the fact that the interfaces are all highly fluctuating, irrespective of the exact geometry, due to the much lower $\mathrm{T}_{\mathrm{g}}$ of the PDMS phase. Noteworthy, for the PI blocks (contrary to PDMS ones) the peak $\alpha$-relaxation time is more representative of the slowest PI segments in the segregated phase since the $\alpha$-relaxation peak is markedly more extended towards high frequencies (see Fig. 6). Consequently, the fast fluctuations of the anchoring points would be the primary reason for the overall speed-up of the PI segmental mobility. This mobility is even higher as the interface is approached.

The temperature dependence of the peak $\alpha$-relaxation times of PI can also be well described by the VFT equation, and again all the parameters, except $T_{0}$, can be fixed to those of the homopolymer. The results of these VFT fittings are summarized in Table 5. As expected, the resulting values for $T_{0}$ and $T_{g B D S}$ for the copolymers are very close to each other within the experimental errors. Nevertheless, they are about 3-4 $\mathrm{K}$ below those of the PI homopolymer. This explains why the peak position in the copolymers matches approximately that of PI homopolymer measured at a temperature $5 \mathrm{~K}$ higher. 


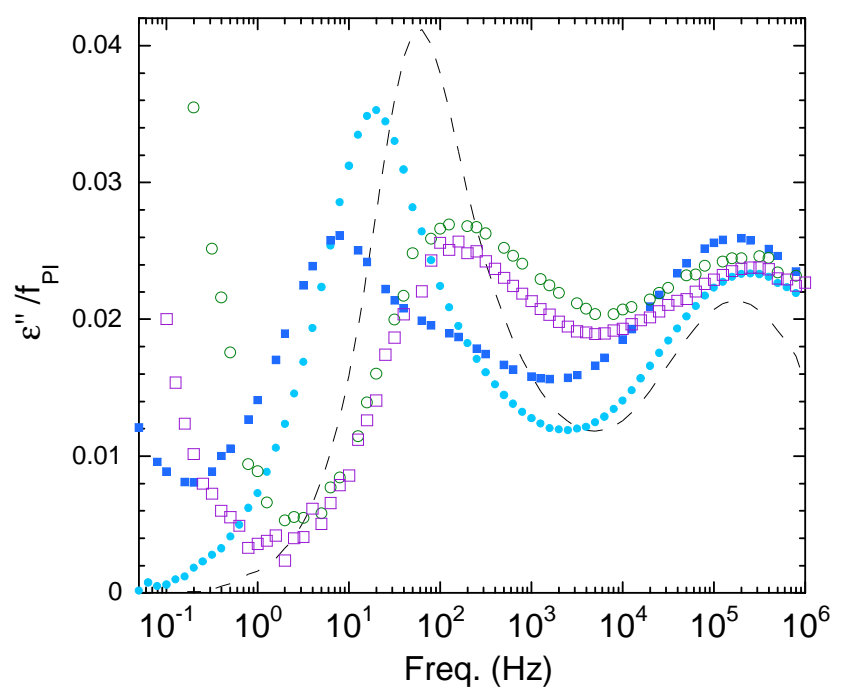

Fig. 7 Dielectric relaxation at $243 \mathrm{~K}$ showing the normal mode and the segmental dynamics of PI for the samples investigated: PI4-PDMS4 (filled squares), PI4-PDMS3.5 (filled circles), PI4-PDMS23 (empty squares), and PI4-PDMS32 (empty circles). The dashed lines correspond to the dielectric relaxation of PI4 at $248 \mathrm{~K}$.

A detailed and quantitative analysis of the shape of the PI $\alpha$-relaxation is only possible for the symmetric copolymers. The reason is two-fold. On one hand, the PI $\alpha$-relaxation of the asymmetric copolymers can only be detected above $230 \mathrm{~K}$, a temperature range where a significant part of the high frequency side of the loss peak is out of the accessible frequency window. On the other hand, the PI component is the minority fraction in the asymmetric copolymers, and consequently, the measured loss data are significantly more prone to noise. Nevertheless, the behavior observed at high temperature is quite similar when comparing the PI $\alpha$-relaxation in symmetric and asymmetric copolymers (see Fig. 7), which suggests a close scenario for all the copolymers. The fits of the loss curves by means of the HN equation (eq. 1) were used to characterize the shape of the peak loss (see lines in Fig. 6). In this analysis, the contribution from the normal mode relaxation at $213 \mathrm{~K}$ has been modeled as a power law and that from PDMS as a flat background. In this way we found that the fits of the $\alpha$-relaxation related to the segmental dynamics of the PI blocks are possible by maintaining the parameter $\alpha_{\mathrm{HN}}$ in eq. 1 fixed to the value obtained for the PI homopolymer (see fitting lines in Fig. 6). This means that the PI $\alpha$-relaxation in the investigated segregated PI phases can be considered as originating from a gradient of mobility such that the majority of PI segments, most likely quite far from the interface, are moving slightly faster than those in pure PI in a rather homogenous manner. However, the long tails towards high frequencies (resulting in 
values of $\gamma_{H N}$ much lower than those found for PI homopolymer) clearly evidence that there are also contributions from much faster PI segments that are more likely located close to the highly fluctuating interface. Thus, here the effect of the interface is opposite to that for PDMS blocks where the high frequency part of the loss peak is the one that remains similar and the effects are more or less prominent at low frequencies (depending on the PDMS volume fraction). The reason for the more heterogeneous PI block segmental dynamics in PI4-PDMS3.5 can be the larger fluctuations of the interface, as expected from the larger amounts of defects observed by SAXS.

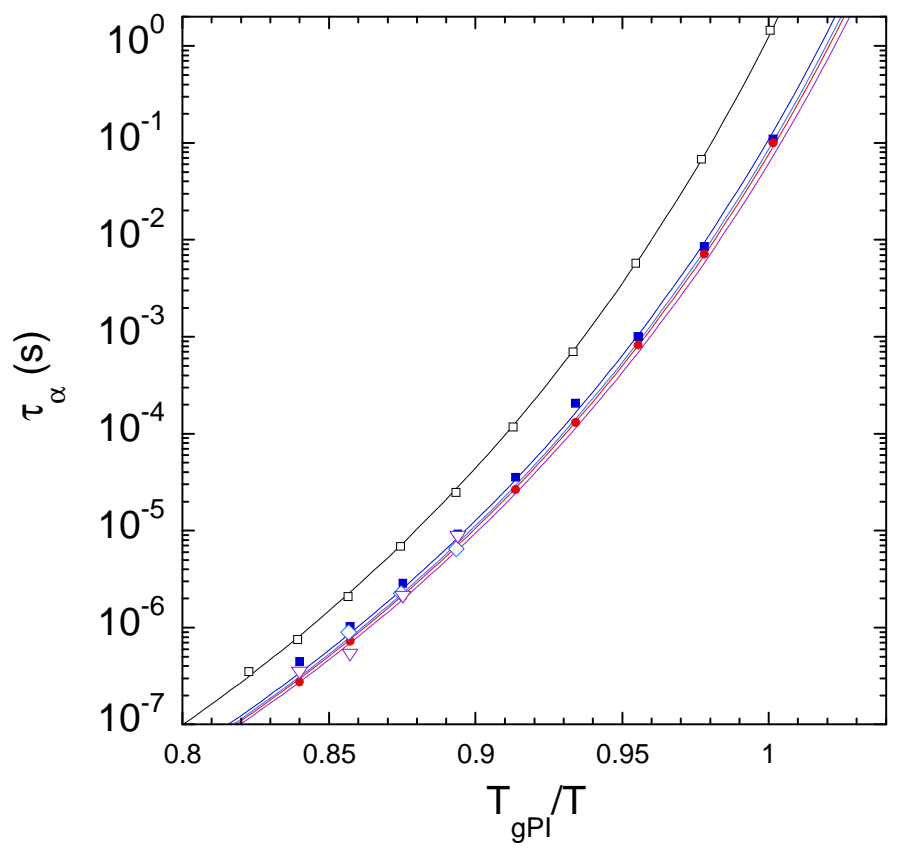

Fig. 8 Temperature dependence of the PI segmental dynamics for the samples investigated: PI4 (squares), PI4-PDMS4 (circles), PI4-PDMS3.5 (squares), PI4-PDMS23 (diamonds), PI4-PDMS32 (inverted triangles). The fitting lines were obtained by using the VFT equation (see text).

The main effect of the temperature on the shape of the $\alpha$-relaxation loss peaks is the reduction of the high frequency broadening, as shown in Fig. 6 for PI4-PDMS4. When considering the dielectric losses at high temperatures, it is apparent that the peak intensity increases and the high frequency behavior becomes steeper, both suggesting that the PI segmental dynamics in the symmetric copolymers becomes more homogeneous by increasing temperature. The corresponding fitting provides values of $\gamma_{H N}$ that increase monotonously with temperature while $\alpha_{H N}$ remains unchanged. These results together with the VFT description of the peak relaxation time shown above, implies again that the broadening of the $\alpha$-relaxation 
of PI blocks respect to that of the homopolymer could be also described in terms of a distribution of $T_{0}$ values.

Table 5 Parameters describing the temperature dependence of the PI relaxation times corresponding to both segmental motions and end-to-end vector fluctuations.

\begin{tabular}{lccccccc}
\hline & $\tau_{\infty \alpha \mathrm{PI}} / \mathrm{s}$ & $\mathrm{D}_{\mathrm{PI}}$ & $\mathrm{T}_{0, \alpha \mathrm{PI}} / \mathrm{K}$ & $\mathrm{T}_{\mathrm{gBDS}} / \mathrm{K}$ & $\tau_{\mathrm{NM}}\left(\mathrm{T}_{\mathrm{g}}\right) / \mathrm{s}$ & $C_{2} / \mathrm{K}$ & $C_{l}$ \\
\hline PI4 & $3.4 \mathrm{e}-14$ & 9.2 & 160.7 & 208.4 & 2570 & 47.9 & 30.2 \\
PI4-PDMS3.5 & $3.4 \mathrm{e}-14(*)$ & $9.2(*)$ & 157.4 & 204.0 & 39.3 & 62.4 & 30.9 \\
PI4-PDMS4 & $3.4 \mathrm{e}-14(*)$ & $9.2(*)$ & 157.7 & 204.5 & 26.0 & 132.3 & 30.3 \\
PI4-PDMS23 & $3.4 \mathrm{e}-14(*)$ & $9.2(*)$ & 157.3 & 203.9 & 45.3 & 62.4 & 27.4 \\
PI4-PDMS32 & $3.4 \mathrm{e}-14(*)$ & $9.2(*)$ & 156.9 & 203.5 & 31.8 & 61.3 & 26.7 \\
\hline
\end{tabular}

$\left.{ }^{*}\right)$ Values fixed in the fitting procedure

Finally, it is also noteworthy to say that the HN fittings performed on the copolymer data provide values of the relaxation strength that, once corrected with the PI volume fraction, are the same than that of PI homopolymer within uncertainties. This suggests that the obtained description captures fully the segmental dynamics of the PI blocks.

\section{Chain Dynamics of PI blocks}

As already mentioned, the normal mode relaxation originated from the fluctuations of the end-to-end PI block vector is well detectable in the experiments at relatively high temperatures $(\mathrm{T}>225 \mathrm{~K})$. Contrary to the PI $\alpha$-relaxation peak, the PI normal mode peak frequency depends significantly on the block copolymer composition (see Fig. 7). Note that all PI blocks have essentially the same molecular weight (see Table 1). By matching the position of the $\alpha$-relaxation peak (i.e. after a shift of $5 \mathrm{~K}$ to higher temperature for PI homopolymer), the PI normal mode peak position of the homopolymer is observed at higher frequencies than those of the symmetric copolymers but at lower frequencies than those of the asymmetric ones. This result shows directly, without any analysis, the quite dramatic effect of the PI segregation geometry on the reorientation of the PI block end-to-end vector.

For temperatures between $230 \mathrm{~K}$ and $310 \mathrm{~K}$, the dielectric loss spectra shift monotonously towards higher frequencies without showing remarkable changes either in shape or in intensity. This is not longer the case at temperatures above $310 \mathrm{~K}$ for PI4-PDMS3.5 and above $370 \mathrm{~K}$ for PI4-PDMS4, but it continues to be the case at all measurable temperatures for the other samples. We recall that at these two temperatures 
the corresponding copolymers exhibit an ODT (see Fig. 2). To explore in detail the changes in the dielectric relaxation, experiments around the respective ODT temperature were performed during a continuous heating at a rate of $1 \mathrm{~K} / \mathrm{min}$. In this way, it was possible to collect the dielectric loss curves every $1 \mathrm{~K}$ and detect clearly the rather abrupt changes of the normal mode relaxations associated to the ODT. The obtained results are shown in Figs. 9a and 9b for PI4-PDMS3.5 and PI4-PDMS4 respectively. In both cases, the rather broad normal mode peak narrows above the ODT temperature to resemble that of PI homopolymer, and the peak position shifts. However, these effects are more dramatic for PI4-PDMS4, in which the peak frequency shifts by nearly one decade. Note that the peak shape in both copolymers above the ODT still remains significantly broader than in the homopolymer, indicative of the remaining heterogeneity likely attributed to the thermal concentration fluctuations in the disordered melt. It is noteworthy that the loss curves in Fig. 9 do not change much when experiments are made crossing the ODT temperature during continuous cooling at $1 \mathrm{~K} / \mathrm{min}$.
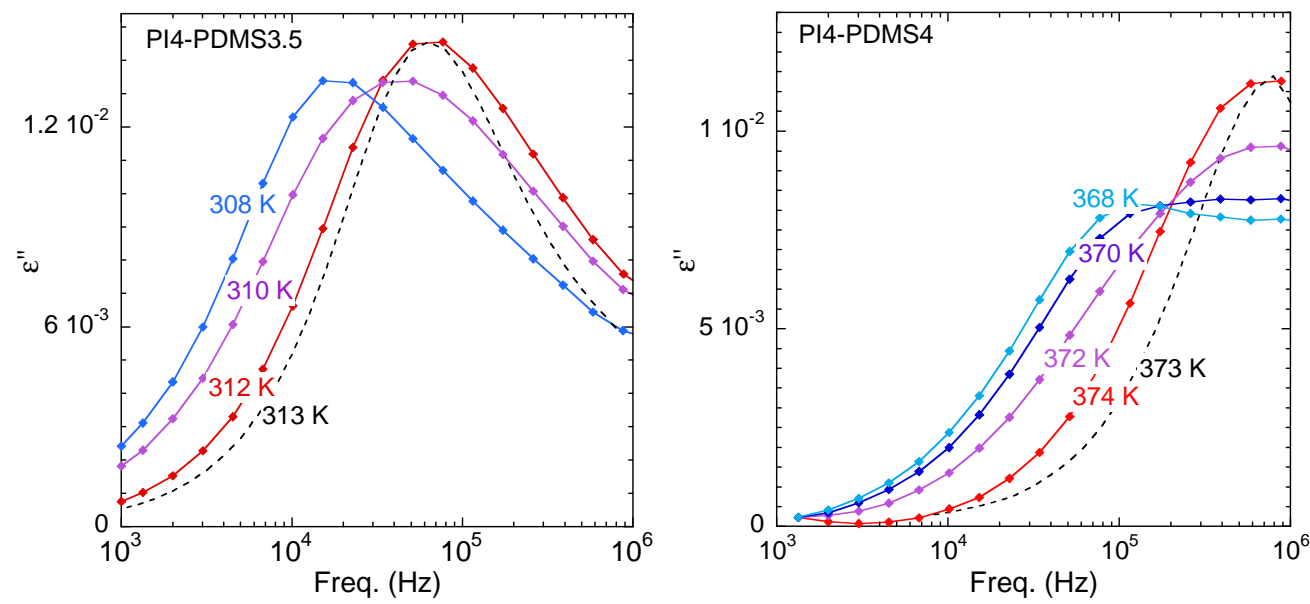

Fig. 9 Evolution of the normal mode relaxation for PI4-PDMS4 (right) and PI4-PDMS3.5 (left) when crossing the ODT range. The dotted lines correspond to the normal mode of PI homopolymer scaled vertically to match the peak intensity.

Fig. 10 presents the times characterizing the PI end-to-end vector dynamics. Chain dynamics theories treat the whole chain motion in terms of the so-called 'Rouse modes' representing the normal modes of motion of a tagged chain subjected to random forces and friction. The latter is characterized by the socalled monomeric friction coefficient [26]. The general approach is that this friction coefficient can be approximately taken as proportional to the segmental relaxation time. Therefore, in the Arrhenius representation used in Fig. 10, the temperature is scaled using the value of $T_{g B D S}$ obtained above, as a way 
to account for the different PI monomeric friction coefficients among the samples. In this way, the apparent effects of the nanostructure on the chain dynamics become clear. There are changes not only in time scales but also in temperature dependence. Block copolymers with cylindrical or spherical segregated PI phases exhibit an end-to-end vector reorientation slightly faster than that of PI homopolymer, but as the temperature decreases the differences in temperature dependence also become very apparent. For the two symmetric copolymers with lamella nanostructure, a slower overall end-to-end vector reorientation with dramatically different temperature dependence is obtained. Only above the ODT temperature, where the lamella nanostructure is lost, time scales and temperature dependencies in these copolymers become closer to those of PI homopolymer.

The temperature dependence of the normal mode can be well described by the Williams-Landel-Ferry (WLF) equation [36], which is formally equivalent to the VFT one but written in terms of the glass transition temperature,

$$
\tau_{N M}(T)=\tau_{N M}\left(T_{g}\right) \exp \left(\frac{C_{1}\left(T-T_{g}\right)}{T-T_{g}+C_{2}}\right) \quad \text { eq. } 4
$$

The values of $C_{1}=30.2$ and $C_{2}=49.1 \mathrm{~K}$ obtained for PI4 homopolymer agree well with those reported in literature [37] for a PI of similar molecular weight.

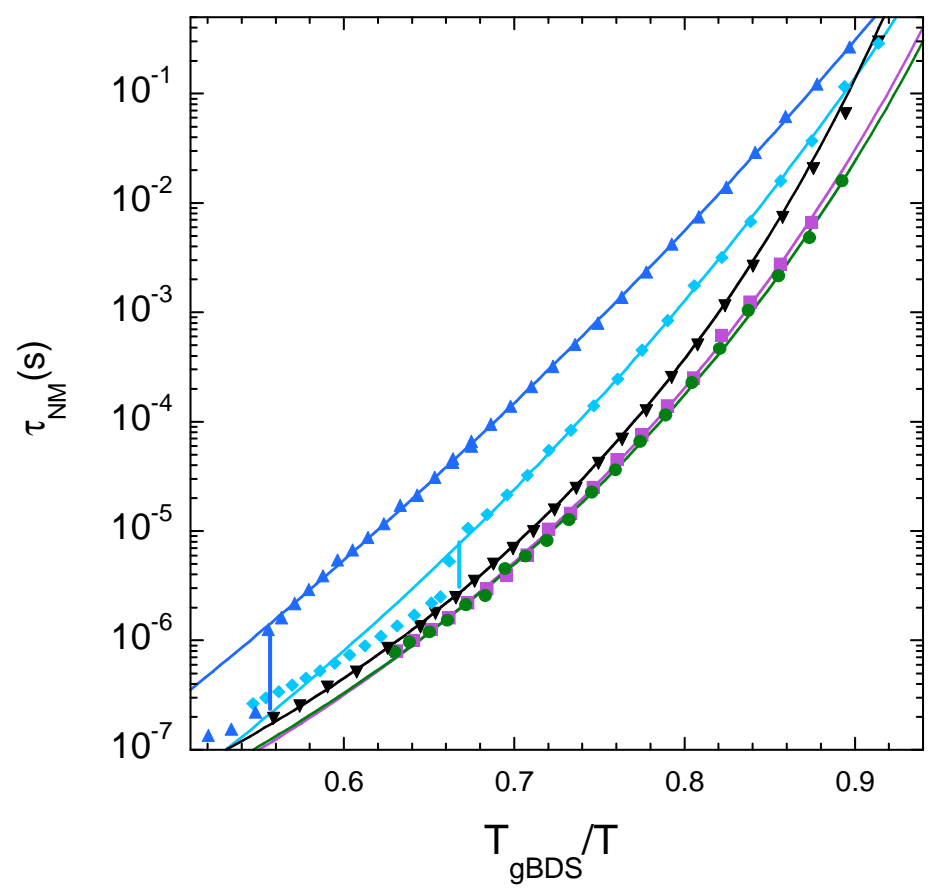

Fig. 10 Temperature dependence of the dielectric normal mode peak times for the samples investigated: PI4 (inverted triangles), PI4-PDMS4 (triangles), PI4-PDMS3.5 (diamonds), PI4-PDMS23 (squares) and 
PI4-PDMS32 (circles). The fitting lines were obtained by using the WLF equation (see text). The vertical lines correspond to the ODT transition detected only for the symmetric copolymers.

The results obtained from the WLF fitting using eq. 4 with the previously reported $T_{g B D S}$ values for the copolymers are included in Table 5. As expected, the parameters obtained for the copolymers are rather different with respect to those of PI homopolymer. This is a clear indication that factors other than the PI internal chain modes affect the end-to-end vector reorientation in the nano-segregated phases. In previous studies, the origin of the PI normal mode dielectric relaxation in PI-PDMS copolymers segregated in cylinders and spheres, both containing nearly the same PI volume fraction $\left(f_{P I} \sim 0.16-0.18\right)$, has been investigated [16,17]. In these works, the crucial role of the diffusion of the anchoring point was recognized. Furthermore, it was demonstrated by neutron scattering experiments [17] that for cylindrically segregated PI phases (as in the case of PI4-PDMS23), the combination of internal chain modes and the diffusion of the anchoring point on the surface of the cylinders (see Fig. 11) provided an excellent description of the end-to-end PI block dynamics. The similarities between the dielectric results for cylindrical and spherical morphologies, shown above and in a previous publication [16], strongly suggest that an equivalent model would be valid for explaining the PI4-PDMS32 normal mode. Within this framework, the acceleration of the normal mode relaxation in the asymmetric copolymers can be directly attributed to the fact that the diffusion of the anchoring point along the interface results in an effective reorientation of the PI block end-to-end vector as schematized in Fig. 11a. Since the PDMS phase surrounding the segregated PI cylinders and spheres has a high mobility, the end-to-end vector reorientation will be faster than in the homopolymer. In addition, it is expected that the temperature dependence of the diffusion coefficient will be simultaneously controlled by the dynamics of both types of segments (PI and PDMS) at the interface [17]. This explains why the temperature dependence of the normal mode time in these symmetric copolymers is weaker than that of the normal mode in PI homopolymer. The situation for the symmetric copolymers with lamella nanostructure is different since the diffusion of the anchoring point in a perfect lamella is not so effective to cause a reorientation of the end-to-end vector (the complete reorientation would be forbidden as schematized in Fig. 11b). However, it has been found that the NM for the lamellas accounts for the complete reorientation of the PI-block end-to-end vector [18]. In fact, the end-to-end vector reorientation process in the lamella phases can be decomposed in two parts. The fastest one is dominated by internal PI modes, as confirmed by molecular 
dynamics simulations [18]. The raw data of PI4-PDMS4 in Fig. 7 show clearly a well-developed component in the high frequency side of the normal mode loss range. The slowest component would be directly controlled by the presence of grain boundaries and diffusing defects along the lamella interface, which would certainly facilitate the complete reorientation of the PI-block end-to-end vector in the lamella phase as schematized in Fig. 11c. So, in these symmetric copolymers the normal mode peak frequency would be representative of this slower component explaining the very different temperature dependence as compared with that of PI homopolymer. The less ordered structure in PI4-PDMS3.5 (see Fig. 1), would also account for the faster normal mode peak time as compared to the better-ordered lamellar structure in PI4-PDMS4.

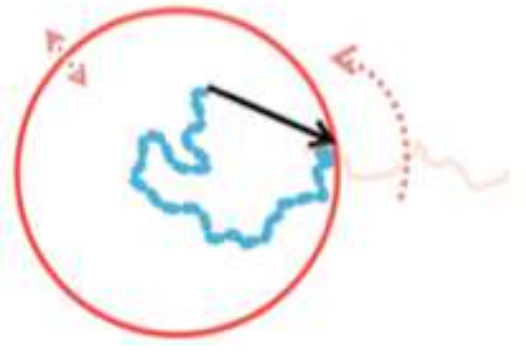

$\mathbf{a}$

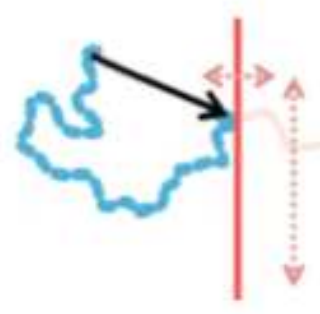

b

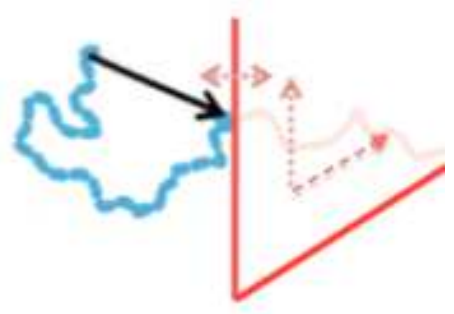

Fig. 11 Schematic plot showing the relevance of the junction point diffusion on the end-to-end vector reorientation for different geometries: a) segregated cylinder or sphere, b) perfect lamella, and c) defective lamella.

Finally, it is worthy of remark that the fast component of the normal mode in the symmetric copolymers reflects the internal chain dynamics of PI block [18]. From the careful deconvolution of the slow and fast components performed in [18], it becomes clear that the internal PI chain dynamics in the segregated lamella phases has a time scale close to that of the whole normal mode in the asymmetric copolymers with cylindrical and spherical PI segregation.

\section{Conclusions}

Dielectric relaxation experiments on PI-PDMS diblock copolymers allowed to investigate the influence of the nanostructure on the segmental dynamics of both components and on the end-to-end vector fluctuations of the PI blocks. The segmental dynamics is sensitive to the soft-hard character of the interface. Not only the shapes of the relaxation curves are clearly modified, but also the peak relaxation 
times are noticeable affected. When the interface is rigid because one component is frozen, the major effect on the other component is a gradual slowing down of the segmental mobility as the interface is approached, but there is also an overall slowing down affecting to most of the segments. Moreover, the segments directly influenced by anchoring to the interface present a distinct contribution evidencing an extremely slow dynamics. On the contrary, when the interface is highly fluctuating there is a gradual speed-up of the segmental mobility of the slower component as the interface is approached, and there is in addition an overall speedup affecting most of the segments. The geometry of the interface seems not to play a major role on the slower component segmental dynamics. For the faster component, the geometry of the interface is not either very relevant, as far as the segregated phase of this component is not completely surrounded by a frozen matrix [30].

On the other hand, the influence of a soft interface is extremely important on the end-to-end chain vector fluctuations. For curved interfaces, the diffusion of the anchoring point facilitates the reorientation of the end-to-end chain vector and the normal mode is detected at frequencies higher than that in the homopolymer. However, for planar interfaces, the completion of the end-to-end chain vector reorientation requires the presence of defects in the lamella structure. Monitoring the changes of the normal mode relaxation upon crossing the ODT range evidences directly the dramatic effect of the lamella nanostructure on the normal mode relaxation.

\section{Acknowledgements}

We warmly acknowledge Dr. Lutz Willner for supplying the block copolymers here investigated. We thank the support of the following research projects MAT2012-31088 supported by the Spanish Ministry "Ministerio de Economía y Competitividad" and IT-654- 13 Supported by the Basque Government. We also acknowledge partial financial support by the European Soft Matter Infrastructure project (ESMI) (Grant Agreement number: 262348).

\section{References}

1. Hadjichristidis N, Pispas S, Floudas G (2003) Block Copolymers: Synthetic Strategies, Physical Properties, and Applications. John Wiley \& Sons, Hoboken, New Jersey

2. Bates FS, Fredrickson GH (1990) Block Copolymer Thermodynamics: Theory and Experiment. Annu Rev Phys Chem 41 (1):525-557

3. Bates FS, Fredrickson GH (1999) Block Copolymers-Designer Soft Materials. Physics Today 52 (2) 
4. Castelletto V, Hamley IW (2007) Block Copolymer Melts. In: Mark J (ed) Physical Properties of Polymers Handbook. Springer New York, pp 641-652

5. Matsen MW, Bates FS (1996) Unifying Weak- and Strong-Segregation Block Copolymer Theories. Macromolecules 29 (4):1091-1098

6. Kirkensgaard JJK, Fragouli P, Hadjichristidis N, Mortensen K (2011) Perforated Lamellae Morphology in Novel P2VP(PDMS-b-PI-b-PS)2 3-Miktoarm Star Quarterpolymer. Macromolecules 44 (3):575582

7. Meuler AJ, Hillmyer MA, Bates FS (2009) Ordered Network Mesostructures in Block Polymer Materials. Macromolecules 42 (19):7221-7250

8. Yamauchi K, Hasegawa H, Hashimoto T, Tanaka H, Motokawa R, Koizumi S (2006) Direct Observation of Polymerization-Reaction-Induced Molecular Self-Assembling Process: In-Situ and Real-Time SANS Measurements during Living Anionic Polymerization of Polyisoprene-blockpolystyrene. Macromolecules 39 (13):4531-4539

9. Mori K, Hasegawa H, Hashimoto T (2001) Order-disorder transition of polystyrene-blockpolyisoprene Part II. Characteristic length as a function of polymer concentration, molecular weight, copolymer composition, and $\chi$ parameter. Polymer 42 (7):3009-3021

10. Kimishima K, Koga T, Hashimoto T (2000) Order-Order Phase Transition between Spherical and Cylindrical Microdomain Structures of Block Copolymer. I. Mechanism of the Transition. Macromolecules 33 (3):968-977

11. Frielinghaus H, Hermsdorf N, Almdal K, Mortensen K, Messé L, Corvazier L, Fairclough JPA, Ryan AJ, Olmsted PD, Hamley IW (2001) Micro- vs. macro-phase separation in binary blends of poly(styrene)-poly(isoprene) and poly(isoprene)-poly(ethylene oxide) diblock copolymers. Europhys Lett $53(5): 680$

12. Almdal K, Mortensen K, Ryan AJ, Bates FS (1996) Order, Disorder, and Composition Fluctuation Effects in Low Molar Mass Hydrocarbon-Poly(dimethylsiloxane) Diblock Copolymers. Macromolecules 29 (18):5940-5947

13. Vigild ME (1997) Mesomorphic Phase Behaviour of Low Molar Mass PEP-PDMS Diblock Copolymers Synthesized by Anionic Polymerization. Ph.D. thesis, Ris $\emptyset$ National Laboratory, Roskilde, Denmark

14. Cochran EW, Morse DC, Bates FS (2003) Design of ABC Triblock Copolymers near the ODT with the Random Phase Approximation. Macromolecules 36 (3):782-792

15. Sanz A, Nogales A, Ezquerra TA (2011) From hard to soft confinement in a symmetric block copolymer: local and segmental dynamics. Soft Matter 7 (14):6477-6483

16. Lund R, Willner L, Alegría A, Colmenero J, Richter D (2008) Self-Concentration and Interfacial Fluctuation Effects on the Local Segmental Dynamics of Nanostructured Diblock Copolymer Melts. Macromolecules 41 (3):511-514

17. Willner L, Lund R, Monkenbusch M, Holderer O, Colmenero J, Richter D (2010) Polymer dynamics under soft confinement in a self-assembled system. Soft Matter 6 (7):1559-1570 
18. Lund R, Barroso-Bujans F, Slimani MZ, Moreno AJ, Willner L, Richter D, Alegría A, Colmenero J (2013) End-to-End Vector Dynamics of Nonentangled Polymers in Lamellar Block Copolymer Melts: The Role of Junction Point Motion. Macromolecules 46 (18):7477-7487

19. Havriliak Jr S, Havriliak SJ (1996) Comparison of the Havriliak-Negami and stretched exponential functions. Polymer 37 (18):4107-4110

20. Kremer F, Schönhals A (2003) Broadband Dielectric Spectroscopy. Springer-Verlag Berlin Heidelberg New York, Germany

21. http://www.chemie.uni-hamburg.de/pc/polymer/software.html]

22. Förster S, Timmann A, Konrad M, Schellbach C, Meyer A, Funari SS, Mulvaney P, Knott R (2005) Scattering Curves of Ordered Mesoscopic Materials. J Phys Chem B 109 (4):1347-1360

23. Chang K, Macosko CW, Morse DC (2007) Ultralow Interfacial Tensions of Polymer/Polymer Interfaces with Diblock Copolymer Surfactants. Macromolecules 40 (10):3819-3830

24. Leibler L (1980) Theory of Microphase Separation in Block Copolymers. Macromolecules 13 (6):1602-1617

25. Fredrickson GH, Helfand E (1987) Fluctuation effects in the theory of microphase separation in block copolymers. J Chem Phys 87 (1):697-705

26. Rubinstein M, Colby RH (2003) Polymer Physics. Oxford University Press, Oxford

27. Rheological measurements were performed in an ARES rotational rheometer, TA Instruments, using an oscillation frequency of $1 \mathrm{~Hz}$, strain amplitude of $2 \%$ and temperature sweep at $3 \mathrm{~K} / \mathrm{min}$. The measuring geometry was plate/plate with a diameter of $25 \mathrm{~mm}$ and a sample thickness of $1 \mathrm{~mm}$. Nitrogen atmosphere was used during the whole experiments.

28. Ding Y, Kisliuk A, Sokolov AP (2003) When Does a Molecule Become a Polymer? Macromolecules 37 (1):161-166

29. Lorthioir C, Alegría A, Colmenero J, Deloche B (2004) Heterogeneity of the Segmental Dynamics of Poly(dimethylsiloxane) in a Diblock Lamellar Mesophase: Dielectric Relaxation Investigations. Macromolecules 37 (20):7808-7817

30. del Valle-Carrandi L, Alegría A, Arbe A, Colmenero J (2011) Unexpected PDMS Behavior in Segregated Cylindrical and Spherical Nanophases of PS-PDMS Asymmetric Diblock Copolymers. Macromolecules 45 (1):491-502

31. Lund R, Alegria A, Goitandia L, Colmenero J, Gonzalez MA, Lindner P (2008) Dynamical and Structural Aspects of the Cold Crystallization of Poly(dimethylsiloxane) (PDMS). Macromolecules 41 (4):1364-1376

32. Vogel H (1921) Das Temperaturabhängigkeitsgesetz der Viskosität von Flüssigkeiten. In: Physik. Zeitschrift, vol 22. pp 645-646

33. Angell CA (1991) Relaxation in liquids, polymers and plastic crystals — strong/fragile patterns and problems. J Non-Cryst Solids 131-133, Part 1:13-31

34. Solar M, Mapesa EU, Kremer F, Binder K, Paul W (2013) The dielectric $\alpha$-relaxation in polymer films: A comparison between experiments and atomistic simulations. Europhys Lett 104 (6):66004

35. Helfand E, Tagami Y (1972) Theory of the Interface between Immiscible Polymers. II. J Chem Phys $56(7): 3592-3601$ 
36. Ferry JD (1980) Viscoelastic properties of polymers. 3rd edn. John Wiley \& Sons, New York

37. Riedel C, Alegría A, Tordjeman P, Colmenero J (2009) Rouse-Model-Based Description of the Dielectric Relaxation of Nonentangled Linear 1,4-cis-Polyisoprene. Macromolecules 42 (21):84928499 